\title{
Simplified Approach for Preliminary Evaluation of Effective Dose Rates for Field Applications of D-T Neutron Generators
}

\author{
Gian Marco Contessa ${ }^{1 *}$, Nadia Cherubini ${ }^{1}$, Giada Gandolfo ${ }^{1}$, Luigi Lepore ${ }^{1}$, Giuseppe A. Marzo ${ }^{1}$, Romolo Remetti $^{2}$ \\ ${ }^{1}$ Italian National Agency for New Technologies, Energy and Sustainable Economic Development, Department of Fusion and \\ Nuclear Safety Technology, Via Enrico Fermi 45, 00044 Frascati (Rome), Italy \\ ${ }^{2}$ Sapienza - University of Rome, Department of Basic and Applied Sciences for Engineering, Via Antonio Scarpa 14, 00161 \\ Rome, Italy
}

Corresponding Author Email: gianmarco.contessa@enea.it

https://doi.org/10.18280/ijsse.110418

Received: 10 January 2021

Accepted: 21 July 2021

\section{Keywords:}

Monte Carlo simulation, neutron dosimeter, neutron generator, occupational safety

\begin{abstract}
Portable neutron generators are one of the most widespread sources of fast neutrons for different applications, from activation analysis to quantitative estimation of fissile and fertile materials in radioactive waste packages. New techniques based on these devices have been developed for homeland security, e.g. neutron interrogations of packages suspected of containing substances such as explosives.

When using portable generators determination of radiation protection quantities is affected by a series of boundary conditions that could differ from an experimental set-up to another, and Radiation Protection Experts cannot assess operator exposure without the aid of time-expensive Monte Carlo simulations. As it is impossible to foresee all kind of scenarios involving such applications, and time is often a critical variable, safety assessment requires faster, even if less accurate, tools for exposure evaluation.

In this article experimental measurements of dose rates in a real scenario involving a D-T neutron generator are considered, to validate the Monte Carlo model developed.

Consequently, it was possible to demonstrate that the calibration factor at $14 \mathrm{MeV}$ for a neutron dosimeter provides conservative dose estimates and to propose a stand-alone, fast, and easy-to-use calculation tool which is usable on field by the Radiation Protection Expert without full Monte Carlo calculations.
\end{abstract}

\section{INTRODUCTION}

Portable neutron generators are one of the most widespread sources of fast neutrons for different kind of analytical applications, ranging from classic activation analysis [1] to the quantitative estimation of the fissile and fertile materials in radioactive waste packages $[2,3]$. Recently, new techniques based on neutron generators have been developed for homeland security, for instance neutron interrogations of containers suspected of containing illicit substances, such as explosives or drugs [4-6].

Current research activities carried out at ENEA involve neutron generators applications for Differential Die Awaytime Analysis (DDAA) [7, 8], fissile and fertile recognition and quantification $[9,10]$, and Fast Neutron Analysis [4, 11] for explosive detection. As such techniques require fast neutrons mainly, the generator is used unshielded, inside an appropriate bunker or, when necessary, in open field situations. Under these conditions, the neutron flux and its energy spectrum at the point of interest is strictly dependent on the generator emission and the surrounding environment.

Neutron radiation field dosimeters are commonly based on the classical Andersson-Braun rem-meter [12]. A thermal neutron detector, typically a proportional counter tube filled with ${ }^{3} \mathrm{He}$, is inserted inside a moderator with material and geometry properly chosen for obtaining a response function which reproduces the variation of the neutron fluence to dose equivalent conversion coefficient against the energy. Original design was based on the old concept of maximum dose equivalent, but the transition to the current ambient dose equivalent $\mathrm{H}^{*}(10)$ did not require substantial modifications [13].

Ideal neutron dosimeters should have a response, per unit dose equivalent, that is independent of neutron energy, but devices currently available are far from being ideal. The ${ }^{3} \mathrm{He}(\mathrm{n}, \mathrm{p})^{3} \mathrm{H}$ cross section varies from about 5400 barn at thermal energies to about 1 barn at $1 \mathrm{MeV}$ and 0.2 barn at 10 $\mathrm{MeV}$. Moreover, as neutron energy increases in the $\mathrm{MeV}$ region, the cross section of the elastic scattering of ${ }^{3} \mathrm{He}$ nuclei becomes more and more important, and corresponding voltage pulses interfere with those ones produced by the $(n, p)$ reaction. In summary, the count rate of uncharacterized neutron fields cannot directly be correlated with neutron dose, unless special detector design and fast electronics, capable to discriminate the ${ }^{3} \mathrm{He}$ recoil pulses, are available. Special application for accelerator facilities have been developed for neutrons up to hundred $\mathrm{MeV}$, as described by Jagerhofer et al. [14, 15] but conventional rem-meters give satisfactory estimation of $\mathrm{H}^{*}(10)$ in the $100 \mathrm{keV}-6 \mathrm{MeV}$ energy range only [13].

A very interesting work by Kim et al. [16] compares six commercial rem-meters, Berthold LB6411, FHT762 WENDI2, NRD-9, SNOOPY NP-2, DINEUTRON, and REM-500, and gives evidence of substantial dose underestimation for neutron energies in the $10 \mathrm{MeV}$ region. 
As a general recommendation, rem-meters should be calibrated in a spectrum which is either similar to the one of the environment of interest or giving at least similar calibration factors [17], but usually the calibration procedures are carried out by putting radionuclide sources such as ${ }^{252} \mathrm{Cf}$, or ${ }^{241} \mathrm{Am}-\mathrm{Be}$ near the detector and minimizing the so-called "room return", i.e. thermal neutrons scattered from the environment and entering the counter. Due to high $(\mathrm{n}, \mathrm{p})$ reaction cross section at thermal energies, room return scattering, in real situations, can significantly affect $H^{*}(10)$ evaluation.

When using commercial rem-counters such as the Berthold LB6411, the user can choose to modify default setting previously obtained by ${ }^{252} \mathrm{Cf}$ calibration simply multiplying the neutron count rate by proper conversion factors, which, obviously, vary with neutron energy. Choosing the right conversion factors requires proper characterization of neutron spectrum at the point of interest, and this is generally obtained by means of preliminary Monte Carlo calculations which consider also the environment where the measurement is performed, so as to account for the room return scattering. Nevertheless, when prompt evaluations are required, as when using D-T neutron generators for homeland security, preliminary Monte Carlo calculations are not suitable. Here, evidence will be provided that conservative evaluation of the ambient dose equivalent $\mathrm{H}^{*}(10)$ can be obtained by using conversion factors at $14 \mathrm{MeV}$ both for open-field situations or confined geometries.

\section{MATERIALS AND METHODS}

The neutron generator considered in this work is the D-T Thermo Scientific MP-320. This system is very compact, portable and light in weight. It is based on a deuterium-tritium sealed source and is designed for laboratory and field applications. It generates a maximum of $10^{8} \mathrm{~s}^{-1}$ neutron emission and is capable of continuous or pulsed output, the latter ranging from $500 \mathrm{~Hz}$ to $20 \mathrm{kHz}$. More details about the device and its capabilities can be found in ref. [18, 19].

The rem-meter Berthold LB6411 consists of a polyethylene (PE) moderator sphere $(250 \mathrm{~mm}$ diameter $)$ with a composite ${ }^{3} \mathrm{He}$ recoil proton proportional counter tube at its center. The instrument is calibrated with the fission spectrum of ${ }^{252} \mathrm{Cf}$ showing a "fluence response" of $1.09 \mathrm{~cm}^{2}$ and $1.27 \mu \mathrm{Sv} \mathrm{h} \mathrm{h}^{-1}$ $\mathrm{cps}^{-1}$ "dose calibration factor". The dose calibration factor is the conversion coefficient between ambient dose equivalent $\mathrm{H}^{*}(10)$ rate and neutron count rate. Table 1 gives its variation vs. energy in the $0.1 \mathrm{MeV}-14 \mathrm{MeV}$ region.

The manufacturer assumes $1.27 \mu \mathrm{Sv} \mathrm{h}^{-1} \mathrm{cps}^{-1}$ cps-to-dose conversion coefficient as default value, whatever the neutron energy spectrum monitored. This is obtained by ${ }^{252} \mathrm{Cf}$ calibration in free air, without any assumption on room return scattering. Consequently, the $\mathrm{H}^{*}(10)$ rate shown on the instrument display is always relative to it. When different neutron spectra are involved, the User's Manual advises the user to calculate the $H^{*}(10)$ rate by multiplying the measured neutron count-rate by a proper conversion coefficient as in Table 1. Listed coefficients cover the region $0.001 \mathrm{eV}-20$ $\mathrm{MeV}[20]$

However, in order to choose the best conversion coefficient, it is necessary to know the actual neutron energy distribution.

Currently, the most efficient method for simulating neutron radiation fields is the Monte Carlo simulation. Monte Carlo approach consists in generating several independent particles and follow each one through all their possible paths (each path is called "particle history"). Then, all the histories are tallied to provide the physical dimension of interest. This is obtained by means of random samplings from all the probability density functions ruling the involved physical phenomena. Very complex geometries can be reproduced, and high accurate results can be obtained. As regards calculation precision, a sufficient computational power should be available for running a statistically significant number of histories within an acceptable time interval. In this work the MCNPX (Monte Carlo N-Particle eXtended) code has been used, in its version MCNPX 2.7.0. An accurate MCNPX model of the MP-320 neutron generator was adopted [21].

Table 1. Variation vs. neutron energy of conversion coefficient between $\mathrm{H}^{*}(10)$ rate and count rate

\begin{tabular}{cccc}
\hline $\begin{array}{c}\text { Energy } \\
{[\mathrm{MeV}]}\end{array}$ & $\begin{array}{c}\text { Dose Calibration } \\
\text { Factor } \\
{\left[\boldsymbol{\mu S v}(\mathbf{h ~ c p s})^{-\mathbf{1}}\right]}\end{array}$ & $\begin{array}{c}\Delta \% \text { respect } \\
\text { to }{ }^{\mathbf{2 5 2}} \mathbf{C f} \\
\mathbf{1 . 2 7} \boldsymbol{\mu S v}(\mathbf{h} \\
\mathbf{c p s})^{-\mathbf{1}}\end{array}$ & $\begin{array}{c}\Delta \% \text { respect to } \\
\mathbf{2 4 1}_{\mathbf{A m}-B e} \\
\mathbf{1 . 2 1} \boldsymbol{\mu S v}(\mathbf{h} \\
\mathbf{c p s})^{-\mathbf{1}}\end{array}$ \\
\hline 0.1 & 1.62 & 27.6 & 33.9 \\
\hline 1.0 & 1.34 & 5.5 & 10.7 \\
\hline 2.0 & 1.06 & -16.5 & -12.4 \\
\hline 5.0 & 1.04 & -18.1 & -14.0 \\
\hline 10.0 & 1.67 & 31.5 & 38.0 \\
\hline 14.0 & 2.46 & 93.7 & 103.3 \\
\hline
\end{tabular}

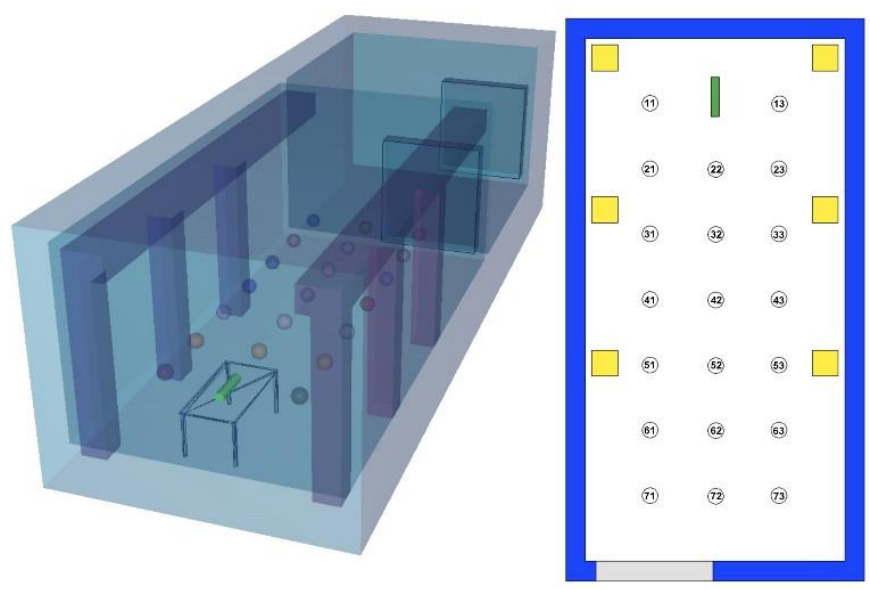

Figure 1. MCNPX-3D-view (left) and MCNPX-horizontal cross-section (right) of the neutron bunker. The neutron generator is in green

In order to test the neutron generator Monte Carlo model, measurements of the neutron emission were carried out in a neutron bunker. The building is shown in Figure 1. The main room is $4 \mathrm{~m}$ large, $8 \mathrm{~m}$ deep and $3 \mathrm{~m}$ high, and the antechamber is $4 \mathrm{~m}$ large, $4 \mathrm{~m}$ deep and $3 \mathrm{~m}$ high. All walls and ceilings are $30 \mathrm{~cm}$ thick concrete slabs, while concrete basement is assumed to be $60 \mathrm{~cm}$ thick, lying on a terrain bank. A metal fence defines a $3 \mathrm{~m}$ respect area from each external walls of the building.

Preliminary to measurements, an accurate model of the building was reproduced in the Monte Carlo simulation. The neutron generator model included in the simulation was derived by Remetti et al. [21]. The simulation of the Berthold LB6411 neutron counter was carried out including its response-curves, taken from the device manual, as multiplicative factors to be used to convert the neutron fluxes in Monte Carlo calculations. 
The Monte Carlo model has been used also for radiation protection purposes and different simulations that have been run to assess the exposures of people in the bunker main room, in the antechamber, and outside.

In such a context, a fundamental phase of the work was the Monte Carlo characterization of the neutron radiation field at different positions inside the bunker main room, to evaluate the "quality" of neutron spectra and choose the adequate cpsto-dose conversion coefficient.

\section{RESULTS}

\subsection{Measurement campaign and Monte Carlo simulations of the bunker}

A measurement campaign has been carried out at specific positions inside a neutron bunker by recording the experimental count rates for 15 seconds live-time; uncertainties have been calculated by applying 1-sigmaPoisson statistics. For each measurement the corresponding MCNPX simulation was carried out.

A $1 \mathrm{~m}$ squared-mesh grid was identified in the bunker main room, and 21 positions have been defined, as shown in Figure 1. Each position is numbered with an $[i, j]$ index, " $i$ " standing for the row, and " $\mathrm{j}$ " for the column. Position [4,2] corresponds to the center of the room.

During a first set of measurements, the neutron generator was maintained at $[i, j]=[1,2]$ position, i.e. the bottom wall of the neutron bunker, and at its minimum power, set at $40 \mathrm{kV}$ accelerator voltage, $20 \mu \mathrm{A}$ deuteron beam, $500 \mathrm{~Hz}$ frequency, and $10 \%$ duty factor, for a minimum neutron yield in $10^{6} \mathrm{~s}^{-1}$.

The rem-counter was sequentially placed at the remaining 20 positions. For each one of the 20 positions, the neutron count rate was measured by Berthold LB6411, and the corresponding MCNPX simulation was carried out.

Table 2 compares, for each $[i, j]$ position, the $H^{*}(10)$ rate obtained by MCNPX calculations, the experimental neutron count rate recorded by the rem-counter, and the $\mathrm{H}^{*}(10)$ rates obtained by multiplying the count rate by the following three different conversion values:

1) default Berthold LB6411 conversion value, $1.27 \mu \mathrm{Sv} \mathrm{h}^{-1}$ $\mathrm{cps}^{-1}$, obtained by ISO - standard ${ }^{252} \mathrm{Cf}$ calibration;

2) $14 \mathrm{MeV}$ conversion value, $2.46 \mu \mathrm{Sv} \mathrm{h}^{-1} \mathrm{cps}^{-1}$, as from the LB6411 manual;

3) MCNPX-weighted count-rate to dose-rate conversion factor, obtained from MCNPX simulated neutron spectrum for each position.

The same data are represented in Figure 2.

It is evident that significant dose rate underestimations could happen when the Berthold LB6411 count-rate to doserate default conversion coefficient is used.

Table 3 and Figure 3 show the results of a second set of measurements obtained by placing the neutron generator at $[\mathrm{i}, \mathrm{j}]$ $=[4,2]$ position, i.e. at the center of the room. Also in this case, significant dose rate underestimations could happen when the Berthold LB6411 count-rate to dose-rate default-value, 1.27 $\mu \mathrm{Sv} \mathrm{h}^{-1} \mathrm{cps}^{-1}$, is used.

In particular, averaged underestimation of about $32 \%$ are obtained for the first set of measurements, and about $36 \%$ for the second one.

The $14 \mathrm{MeV}$ conversion coefficient, $2.46 \mu \mathrm{Sv} \mathrm{h}^{-1} \mathrm{cps}^{-1}$, produces conservative results at each position, overestimating of about $31 \%$ for the first set of measurements and $24 \%$ for the second one. In both cases the MCNPX-weighted coefficient produces both underestimations or overestimations, the averaged deviation being about $1 \%$.

Table 2. Dose rates calculated with the MCNPX model for the neutron bunker vs. experimental values (neutron generator near a wall)

\begin{tabular}{|c|c|c|c|c|c|c|}
\hline $\begin{array}{c}\text { Probe } \\
\text { position } \\
{[\mathbf{i} ; \mathbf{j}]}\end{array}$ & $\begin{array}{c}\text { MCNPX } \\
\text { calculated } \\
\mathbf{H}^{*}(10)\left[\mu S v h^{-}\right. \\
1]\end{array}$ & $\begin{array}{c}\text { Measured } \\
\text { count rate } \\
{[\mathrm{cps}]}\end{array}$ & $\begin{array}{c}\mathrm{H}^{*}(10) \text { with } \\
\text { default conversion } \\
\text { coefficient }{ }^{252} \mathrm{Cf} \\
{\left[\mu \mathrm{Sv} \mathrm{h}^{-1}\right]} \\
\end{array}$ & $\begin{array}{c}H^{*}(10) \text { with } 14 \\
\text { MeV conversion } \\
\text { coefficient }\left[\mu S v h^{-}\right. \\
1]\end{array}$ & $\begin{array}{l}\text { MCNPX-weighted } \\
\text { conversion } \\
\text { coefficient }[\mu S v(h \\
\left.\text { cps) })^{-1}\right]\end{array}$ & $\begin{array}{c}H^{*}(10) \text { with } \\
\text { MCNPX-weighted } \\
\text { conversion } \\
\text { coefficient }\left[\mu S v h^{-1}\right]\end{array}$ \\
\hline$[1,1]$ & 32.8 & 13.4 & $17.0( \pm 7 \%)$ & $33.0( \pm 7 \%)$ & 2.17 & $29.1( \pm 7 \%)$ \\
\hline$[1,2]$ & \multicolumn{6}{|c|}{ neutron generator position } \\
\hline$[1,3]$ & 34.0 & 14.1 & $17.9( \pm 7 \%)$ & $34.6( \pm 7 \%)$ & 2.23 & $31.4( \pm 7 \%)$ \\
\hline$[2,1]$ & 28.6 & 13.7 & $17.4( \pm 7 \%)$ & $33.6( \pm 7 \%)$ & 2.06 & $28.2( \pm 7 \%)$ \\
\hline$[2,2]$ & 55.6 & 28.0 & $35.6( \pm 5 \%)$ & $68.9( \pm 5 \%)$ & 2.22 & $62.2( \pm 5 \%)$ \\
\hline$[2,3]$ & 29.6 & 11.8 & $15.0( \pm 8 \%)$ & $29.1( \pm 8 \%)$ & 2.13 & $25.2( \pm 8 \%)$ \\
\hline$[3,1]$ & 13.3 & 8.3 & $10.5( \pm 9 \%)$ & $20.4( \pm 9 \%)$ & 1.90 & $16.7( \pm 9 \%)$ \\
\hline$[3,2]$ & 15.7 & 7.1 & $9.0( \pm 10 \%)$ & $17.4( \pm 10 \%)$ & 1.96 & $13.9( \pm 10 \%)$ \\
\hline$[3,3]$ & 13.5 & 8.5 & $10.8( \pm 9 \%)$ & $21.0( \pm 9 \%)$ & 1.93 & $16.5( \pm 9 \%)$ \\
\hline$[4,1]$ & 7.5 & 5.1 & $6.5( \pm 11 \%)$ & $12.5( \pm 11 \%)$ & 1.80 & $9.2( \pm 11 \%)$ \\
\hline$[4,2]$ & 8.1 & 4.7 & $6.0( \pm 12 \%)$ & $11.6( \pm 12 \%)$ & 1.83 & $8.6( \pm 12 \%)$ \\
\hline$[4,3]$ & 7.5 & 3.7 & $4.7( \pm 13 \%)$ & $9.1( \pm 13 \%)$ & 1.80 & $6.7( \pm 13 \%)$ \\
\hline$[5,1]$ & 4.8 & 2.7 & $3.5( \pm 16 \%)$ & $6.7( \pm 16 \%)$ & 1.75 & $4.8( \pm 16 \%)$ \\
\hline$[5,2]$ & 5.0 & 2.3 & $3.0( \pm 17 \%)$ & $5.8( \pm 17 \%)$ & 1.77 & $4.1( \pm 17 \%)$ \\
\hline$[5,3]$ & 4.8 & 2.1 & $2.7( \pm 18 \%)$ & $5.3( \pm 18 \%)$ & 1.76 & $3.8( \pm 18 \%)$ \\
\hline$[6,1]$ & 3.4 & 2.3 & $2.9( \pm 17 \%)$ & $5.6( \pm 17 \%)$ & 1.73 & $4.0( \pm 17 \%)$ \\
\hline$[6,2]$ & 3.5 & 2.3 & $3.0( \pm 17 \%)$ & $5.8( \pm 17 \%)$ & 1.73 & $4.0( \pm 17 \%)$ \\
\hline$[6,3]$ & 3.4 & 2.0 & $2.5( \pm 18 \%)$ & $4.9( \pm 18 \%)$ & 1.73 & $3.4( \pm 18 \%)$ \\
\hline$[7,1]$ & 2.7 & 1.0 & $1.4( \pm 25 \%)$ & $2.7( \pm 25 \%)$ & 1.69 & $1.8( \pm 25 \%)$ \\
\hline$[7,2]$ & 2.7 & 2.0 & $2.5( \pm 18 \%)$ & $4.8( \pm 18 \%)$ & 1.69 & $3.3( \pm 18 \%)$ \\
\hline$[7,3]$ & 2.6 & 1.2 & $1.5( \pm 24 \%)$ & $3.0( \pm 24 \%)$ & 1.70 & $2.0( \pm 24 \%)$ \\
\hline
\end{tabular}




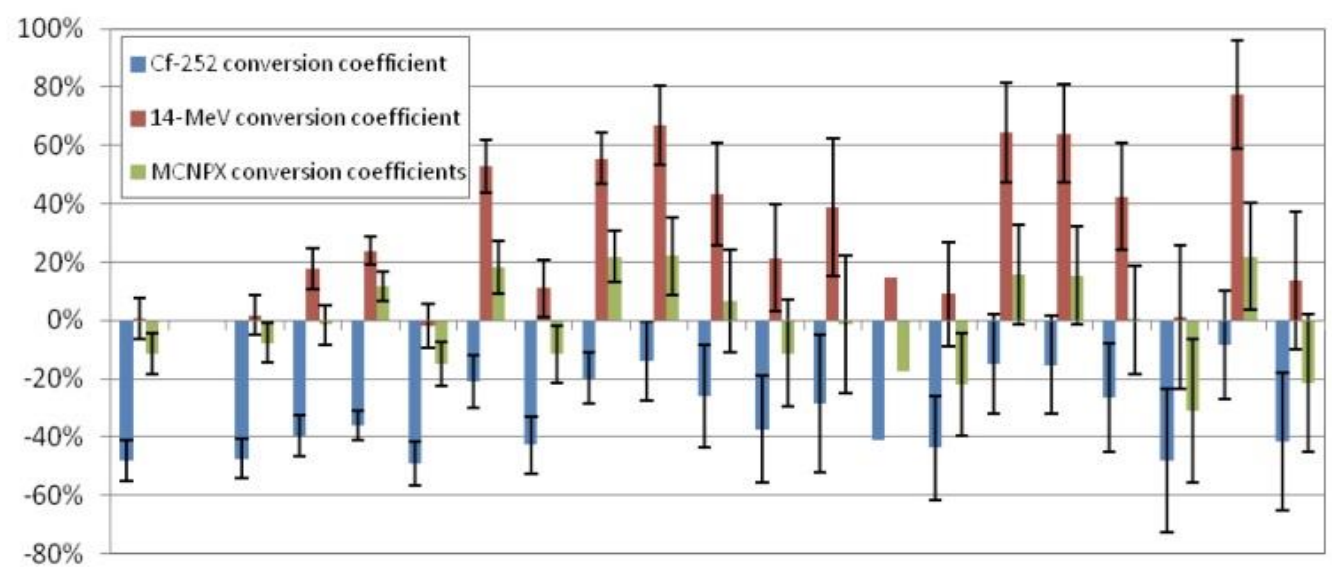

[11] [12] [13] [21] [22] [23] [31] [32] [33] [41] [42] [43] [51] [52] [53] [61] [62] [63] [71] [72] [73]

Figure 2. Neutron generator near a wall. Deviations from Berthold LB6411's dose rates vs. MCNPX dose rates

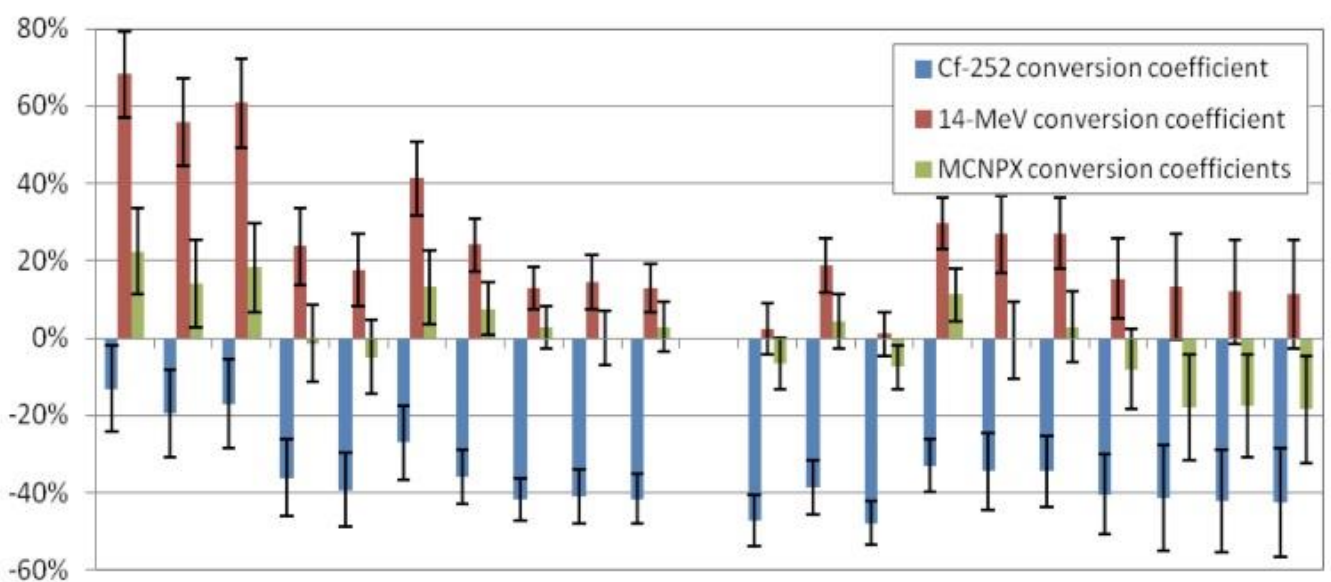

[11] [12] [13] [21] [22] [23] [31] [32] [33] [41] [42] [43] [51] [52] [53] [61] [62] [63] [71] [72] [73]

Figure 3. Neutron generator at the center of the room. Deviations from Berthold's dose rates vs. MCNPX dose rates

Table 3. Dose rate calculated with MCNPX model for the bunker vs. experimental values (neutron generator at center)

\begin{tabular}{|c|c|c|c|c|c|c|}
\hline $\begin{array}{c}\text { Probe } \\
\text { position } \\
{[\mathrm{i} ; \mathrm{j}]}\end{array}$ & $\begin{array}{c}\text { MCNPX } \\
\text { calculated } \\
H^{*}(10)\left[\mu S v h^{-}\right. \\
1]\end{array}$ & $\begin{array}{c}\text { Measured } \\
\text { count rate } \\
{[\mathrm{cps}]}\end{array}$ & $\begin{array}{c}\mathrm{H}^{*}(10) \text { with } \\
\text { default conversion } \\
\text { coefficient }{ }^{252} \mathrm{Cf} \\
{\left[\mu \mathrm{Sv} \mathbf{h}^{-1}\right]}\end{array}$ & $\begin{array}{c}H^{*}(10) \text { with } 14 \\
\text { MeV conversion } \\
\text { coefficient }\left[\mu S v h^{-}\right. \\
1]\end{array}$ & $\begin{array}{l}\text { MCNPX-weighted } \\
\text { conversion } \\
\text { coefficient }[\mu S v(h \\
\left.\text { cps })^{-1}\right]\end{array}$ & $\begin{array}{c}\mathbf{H}^{*}(10) \text { with } \\
\text { MCNPX-weighted } \\
\text { conversion } \\
\text { coefficient }\left[\mu S v h^{-1}\right]\end{array}$ \\
\hline$[1,1]$ & 7.9 & 5.4 & $6.9( \pm 11 \%)$ & $13.3( \pm 11 \%)$ & 1.79 & $9.7( \pm 11 \%)$ \\
\hline$[1,2]$ & 8.2 & 5.2 & $6.6( \pm 11 \%)$ & $12.8( \pm 11 \%)$ & 1.80 & $9.4( \pm 11 \%)$ \\
\hline$[1,3]$ & 7.7 & 5.0 & $6.4( \pm 12 \%)$ & $12.4( \pm 12 \%)$ & 1.81 & $9.1( \pm 12 \%)$ \\
\hline$[2,1]$ & 13.3 & 6.7 & $8.5( \pm 10 \%)$ & $16.4( \pm 10 \%)$ & 1.96 & $13.1( \pm 10 \%)$ \\
\hline$[2,2]$ & 15.3 & 7.3 & $9.3( \pm 10 \%)$ & $18.0( \pm 10 \%)$ & 1.99 & $14.6( \pm 10 \%)$ \\
\hline$[2,3]$ & 13.0 & 7.5 & $9.5( \pm 9 \%)$ & $18.4( \pm 9 \%)$ & 1.97 & $14.7( \pm 9 \%)$ \\
\hline$[3,1]$ & 28.1 & 14.2 & $18.0( \pm 7 \%)$ & $34.9( \pm 7 \%)$ & 2.13 & $30.2( \pm 7 \%)$ \\
\hline$[3,2]$ & 50.6 & 23.2 & $29.5( \pm 5 \%)$ & $57.1( \pm 5 \%)$ & 2.24 & $52.0( \pm 5 \%)$ \\
\hline$[3,3]$ & 28.4 & 13.2 & $16.8( \pm 7 \%)$ & $32.5( \pm 7 \%)$ & 2.15 & $28.4( \pm 7 \%)$ \\
\hline$[4,1]$ & 35.7 & 16.4 & $20.8( \pm 6 \%)$ & $40.3( \pm 6 \%)$ & 2.24 & $36.7( \pm 6 \%)$ \\
\hline$[4,2]$ & \multicolumn{6}{|c|}{ neutron generator position } \\
\hline$[4,3]$ & 36.1 & 15.0 & $19.1( \pm 7 \%)$ & $36.9( \pm 7 \%)$ & 2.25 & $33.8( \pm 7 \%)$ \\
\hline$[5,1]$ & 28.4 & 13.7 & $17.4( \pm 7 \%)$ & $33.7( \pm 7 \%)$ & 2.16 & $29.6( \pm 7 \%)$ \\
\hline$[5,2]$ & 50.4 & 20.7 & $26.3( \pm 6 \%)$ & $50.9( \pm 6 \%)$ & 2.25 & $46.6( \pm 6 \%)$ \\
\hline$[5,3]$ & 27.7 & 14.6 & $18.5( \pm 7 \%)$ & $35.9( \pm 7 \%)$ & 2.11 & $30.8( \pm 7 \%)$ \\
\hline$[6,1]$ & 13.0 & 6.7 & $8.5( \pm 10 \%)$ & $16.5( \pm 10 \%)$ & 1.93 & $12.9( \pm 10 \%)$ \\
\hline$[6,2]$ & 15.3 & 7.9 & $10.0( \pm 9 \%)$ & $19.4( \pm 9 \%)$ & 1.99 & $15.7( \pm 9 \%)$ \\
\hline$[6,3]$ & 13.3 & 6.2 & $7.9( \pm 10 \%)$ & $15.3( \pm 10 \%)$ & 1.96 & $12.2( \pm 10 \%)$ \\
\hline$[7,1]$ & 7.7 & 3.5 & $4.5( \pm 14 \%)$ & $8.7( \pm 14 \%)$ & 1.78 & $6.3( \pm 14 \%)$ \\
\hline$[7,2]$ & 8.2 & 3.7 & $4.7( \pm 13 \%)$ & $9.2( \pm 13 \%)$ & 1.81 & $6.8( \pm 13 \%)$ \\
\hline$[7,3]$ & 7.5 & 3.4 & $4.3( \pm 14 \%)$ & $8.4( \pm 14 \%)$ & 1.80 & $6.1( \pm 14 \%)$ \\
\hline
\end{tabular}




\subsection{Simulated building scenarios}

Having found a good agreement between experimental and simulated results, MCNPX was also utilized as a tool for radiation protection considerations inside two typical industrial buildings of larger dimensions than those of the bunker, which can be potential scenarios for homeland security applications.

Hence, simulations of two typical industrial buildings have been conducted for homeland security applications:

a) large bunker: $6.6 \mathrm{~m}(\mathrm{~L}) \times 6.6 \mathrm{~m}(\mathrm{~W}) \times 6.6 \mathrm{~m}(\mathrm{H}), 30 \mathrm{~cm}$ thick concrete walls;

b) very large bunker: $12.6 \mathrm{~m}(\mathrm{~L}) \times 12.6 \mathrm{~m}(\mathrm{~W})$ x $12.6 \mathrm{~m}(\mathrm{H})$, $30 \mathrm{~cm}$ thick concrete walls.

Four sets of simulations have been carried out by combining those two geometries with two different positions for the neutron generator, one at room-center, the other near a perimetric wall. In both geometries considered, $1 \mathrm{~m}$ squaredmesh grid has been used, with 48 and 168 positions evaluated respectively, the "central value" of this matrix corresponding to the geometrical center of the room considered.
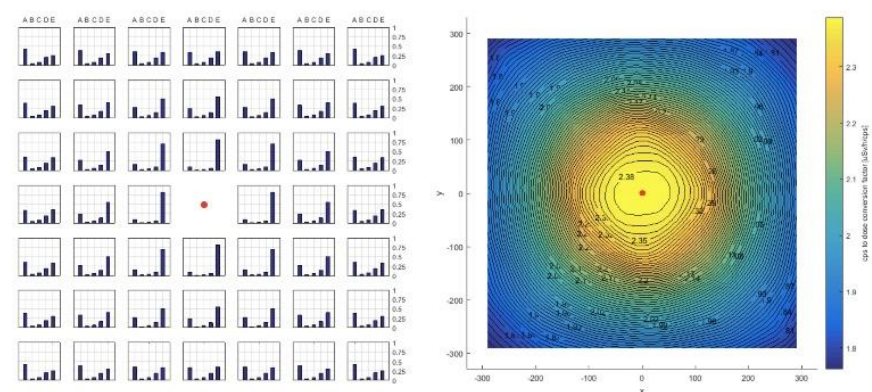

Figure 4. Large bunker: conversion coefficient vs position; neutron generator (red dot) at center. Simulated neutron spectra vs. spatial position (left) and MCNPX weighted count-rate to dose-rate conversion factor (right)

Moreover, at each position, the response of the Berthold LB6411 detector was simulated, and the neutron spectrum and the MCNPX-weighted count-rate to dose-rate conversion coefficient for LB6411 have been evaluated for radiation protection purposes.

The Berthold LB6411 count-rate to dose-rate conversion coefficients vs. neutron energy from the manufacturer has been used as multiplier in the appropriate function of the Monte Carlo input deck. Results are reported in Figures 4 - 7.

Simulated neutron spectra are shown at each position of the $1 \mathrm{~m}$ squared-mesh grid point, and are regrouped in the following neutron energy windows:

- A, $1 \mathrm{E}-9 \div 1 \mathrm{E}-6 \mathrm{MeV}$;

- $\quad$ B, $1 \mathrm{E}-6 \div 1 \mathrm{E}-4 \mathrm{MeV}$;

- $\quad$ C, $1 \mathrm{E}-4 \div 1 \mathrm{E}-1 \mathrm{MeV}$;

- $\quad \mathrm{D}, 1 \mathrm{E}-1 \div 1 \mathrm{E}+1 \mathrm{MeV}$;

- $\mathrm{E}, 1 \mathrm{E}+1 \div 1.5 \mathrm{E}+1 \mathrm{MeV}$.

For both large and very large buildings, the highest value of the simulated weight for count-rate to dose-rate conversion coefficients is obtained when the neutron generator is placed at the center of the room.

This is due to the minor importance of scattering phenomena (Figure 4 and Figure 6) with respect to the case when the neutron generator is near the wall (Figure 5 and Figure 7). When neutron spectra low-tails dominate with respect to $14 \mathrm{MeV}$, i.e. when a significant "room return" is present, conversion coefficients assume lower values; when the 10 to $15 \mathrm{MeV}$ neutrons-bin dominates, conversion coefficients assume higher values.
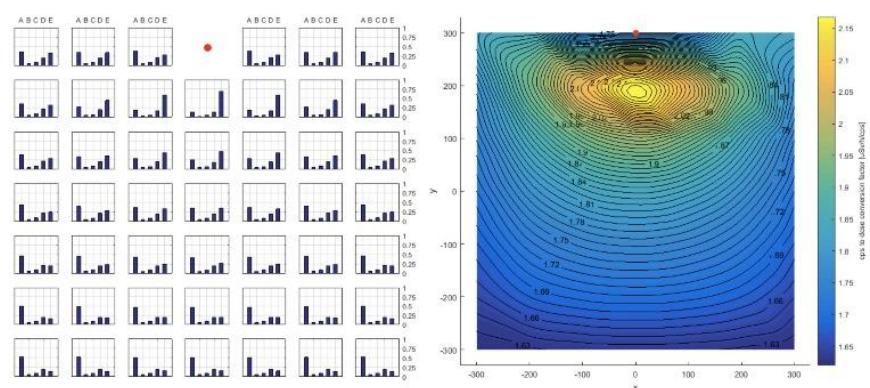

Figure 5. Large bunker: conversion coefficient vs position; neutron generator (red dot) near a wall. Simulated neutron spectra vs. spatial position (left) and MCNPX weighted count-rate to dose-rate conversion factor (right)
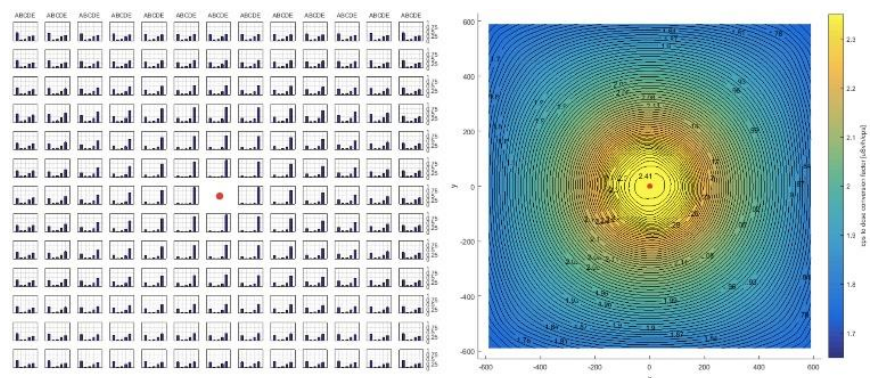

Figure 6. Very large bunker: conversion coefficient vs position; neutron generator (red dot) at center. Simulated neutron spectra vs. spatial position (left) and MCNPX weighted count-rate to dose-rate conversion factor (right)

However, whatever the position considered, the MCNPXweighted count-rate to dose-rate conversion coefficients appear higher than the $1.27 \mu \mathrm{Sv} \mathrm{h} \mathrm{cps}^{-1}$ default value (corresponding to the ${ }^{252} \mathrm{Cf}$ self-fission ISO-neutron spectrum), and lower than the $2.46 \mu \mathrm{Sv} \mathrm{h}^{-1} \mathrm{cps}^{-1}$ value (corresponding to the hypothesis of monoenergetic $14 \mathrm{MeV}$ neutrons).

In summary, in both experimental campaigns, and in the four simulated test cases, the Radiation Protection Expert could determine the $\mathrm{H}^{*}(10)$ by the Berthold LB6411 remcounter by assuming, as a conservative hypothesis, the 14 $\mathrm{MeV}$ count-rate to dose-rate conversion coefficient.
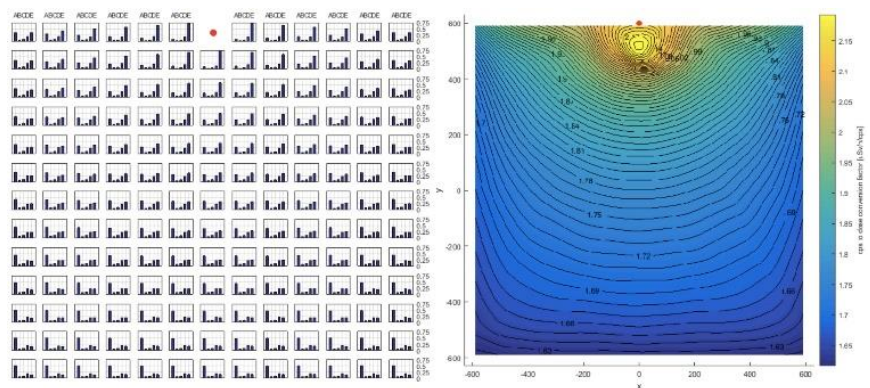

Figure 7. Very large bunker: conversion coefficient vs. position; neutron generator (red dot) near a wall. Simulated neutron spectra vs. spatial position (left) and MCNPX weighted count-rate to dose-rate conversion factor (right) 


\section{DISCUSSION}

\subsection{Derived calculation tool based on Monte Carlo simulations}

As shown in Figures 4-7, the neutron spectrum at the point of interest where the exposure has to be evaluated is strictly dependent on the distance from the source point, and on scattering phenomena caused by moderating structures that may be present in the real-life scenario. Here are the results used forward for radiation protection purposes:

a) $14 \mathrm{MeV}$ component of neutron spectrum vs. "scatteredneutrons". The results of the theoretical simulations (Figures 4-7) show that the $14 \mathrm{MeV}$ "direct-component", namely the energy range " $\mathrm{E}$ " $(10 \div 15 \mathrm{MeV})$, is never less than $25 \%$ of the total; on the contrary, the "scatteredcomponent", namely the energy range $\mathrm{A}+\mathrm{B}+\mathrm{C}+\mathrm{D}(1 \mathrm{E}-9$ $\div 1 \mathrm{E}+1 \mathrm{MeV}$ ), reaches $75 \%$ at maximum. With these premises, if the "direct-component" neutron fluence could be determined by analytical calculation with the hypothesis of spherical propagation of $14 \mathrm{MeV}$ neutrons from the source, the "scattered-component" neutron fluence is at maximum 3 times the "direct-component" neutron fluence according to simulations. For conservative evaluation, and considering that the "scattered-component" could be enhanced due to the presence of objects and materials in the scenario considered (as in the performed simulations buildings were empty), the "scattered-component" neutron fluence can be assumed 10 times the "direct-component" neutron fluence, instead of 3 .

b) Conversion factor from neutron flux to effective dose rate. As known, the "direct-component" $(14.1 \mathrm{MeV})$ in the neutron spectrum should be converted to effective dose

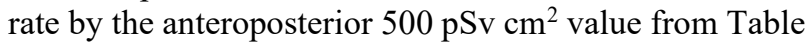
A.5 of ICRP Publication 116 [22]; the "scattered-neutrons" conversion value to dose rate should be evaluated with the lower energies values of the same table, once neutron spectrum is known or estimated by former Monte Carlo simulations. Supposing that simulations or further considerations on the "scattered-neutrons" are not

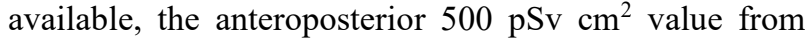
ICRP Publication 116 (the maximum, namely, as it considers the worst-case exposure condition) can be assumed as a conservative conversion also for the low energy tail of the neutron spectrum.

Formally, the proposed approach can be summarized in the equation below. The effective dose rate, $\dot{\mathrm{E}}$, can be split into the $14 \mathrm{MeV}$ "direct-component" contribution (DC), plus the "scattered-component" contribution (SC) due to "scatteredneutrons":

$$
\dot{\mathrm{E}}=\frac{\dot{\mathrm{N}}}{4 \pi \mathrm{R}^{2}} \cdot \mathrm{CF}_{\mathrm{DC}}+\mathrm{SC} \cdot \mathrm{CF}_{\mathrm{SC}}
$$

where:

- $\quad \dot{\mathrm{E}}$ : effective dose rate $\left[\mathrm{pSv} \mathrm{s}^{-1}\right]$;

- $\quad \dot{\mathrm{N}}$ : neutron emission by the neutron generator $\left[\mathrm{s}^{-1}\right]$;

- $\mathrm{R}$ : distance from the neutron generator target to the evaluation point $[\mathrm{cm}]$;

- $\mathrm{CF}_{\mathrm{DC}}$ : "direct-component" conversion factor to

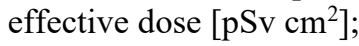

- SC: "scattered component" $\left[\begin{array}{cc}\mathrm{cm}^{-2} & \mathrm{~s}^{-1}\end{array}\right]$; for a conservative approach, $\mathrm{SC}=10 \mathrm{~N} / 4 \mathrm{R}^{2}$ is assumed;
- $\mathrm{CF}_{\mathrm{SC}}$ : "scattered component" conversion factor to

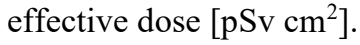

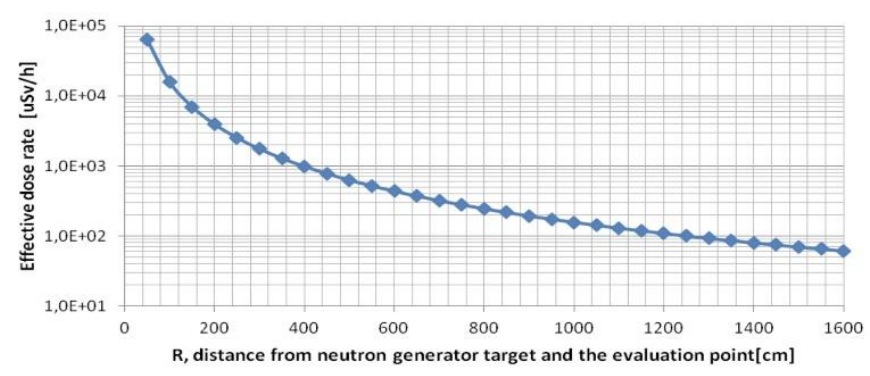

Figure 8. Effective dose rate from Eq. (2) vs. distance R between the neutron generator target and the evaluation point (neutron yield: $10^{8} \mathrm{~s}^{-1}$ )

According to previous assumptions, and having assumed conservatively $\mathrm{CF}_{\mathrm{DC}}=\mathrm{CF}_{\mathrm{SC}}=500 \mathrm{pSv} \mathrm{cm}{ }^{2}$, Eq. (1) can be reformulated as Eq. (2), giving the effective dose rate related to the neutron emission $\dot{\mathrm{N}}$ and the distance between the neutron generator and the point of interest R. A factor of $3600 / 10^{6}$ is applied to convert $\dot{\mathrm{E}}\left[\mathrm{pSv} \mathrm{s}^{-1}\right]$ in $\dot{\mathrm{E}}\left[\mathrm{Sv} \mathrm{s}{ }^{-1}\right]$.

$$
\dot{\mathrm{E}}=\left(\frac{\dot{\mathrm{N}}}{4 \pi \mathrm{R}^{2}}+10 \frac{\dot{\mathrm{N}}}{4 \pi \mathrm{R}^{2}}\right) \cdot 500 \cdot \frac{3600}{10^{6}}=19.8 \frac{\dot{\mathrm{N}}}{4 \pi \mathrm{R}^{2}}
$$

In Figure 8 the graphical representation of Eq. (2), $\dot{E}$ vs. R, is shown, considering $\dot{N}=10^{8} \mathrm{~s}^{-1}$ emission from the neutron generator.

\subsection{Exposure evaluations}

Calculations presented hereafter are intended to give the order of magnitude of the radiation protection quantities of interest, and to demonstrate the conservativeness of the approach. Both bunker and open field cases are discussed.

\subsubsection{Exposure evaluations in the bunker scenario}

A bunker scenario is here considered where a D-T neutron generator is operated. The case study involves operations in controlled environments using a neutron generator, e.g. for laboratory applications. Geometries, materials, surrounding structures, etc., are known a priori: conservative assumptions of the general approach in Eq. (2) can be removed and a more accurate evaluation of the specific case can be done.

The "bunker" here considered is the same discussed in Figure 1. Specific information about the layout of the bunker is reported in Figure 9.

The bunker has been reproduced in the MCNPX code for evaluating effective dose rates outside the building. It should be noted that simulations are run considering the main room and the antechamber as empty spaces, without any presence of further shielding material. Results are reported in Figure 10, assuming the maximum neutron generator yield of $10^{8} \mathrm{~s}^{-1}$.

Such a case study, and its results in Figures 10 and 11, can be useful to estimate the conservativeness of the approach proposed by Eq. (2). Figure 11 reports the effective dose rates outside the bunker, calculated by Eq. (2) without considering the shielding effects of the concrete walls. As expected, dose rates are overestimated in comparison with values from MCNPX simulation (shown in Figure 10) which, on the contrary, account for the presence of shielding walls. Dose rates are overestimated by two orders of magnitude, showing 
that the shielding effect of a single $30 \mathrm{~cm}$ thick concrete slab reduces effective dose rates by a factor of 50 at least, when using the approach in Eq. (2).

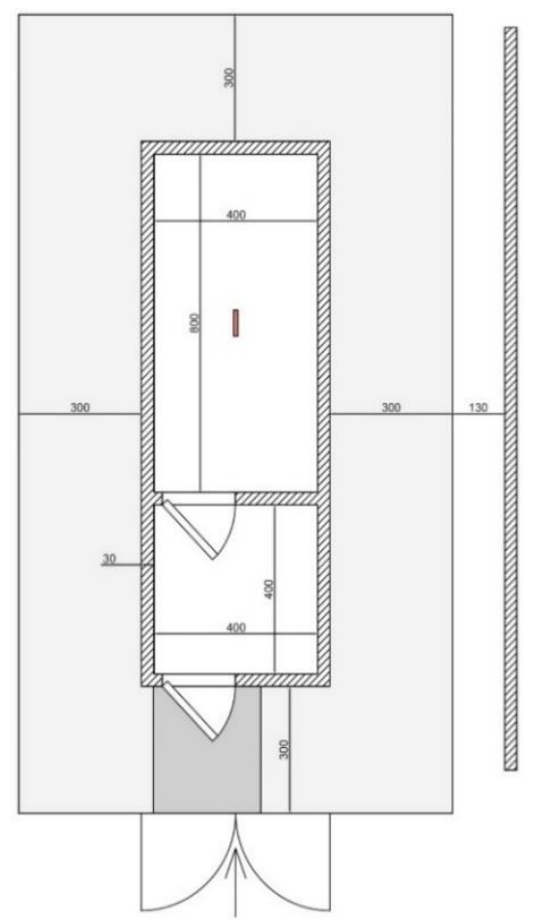

Figure 9. Geometry of the neutron bunker analyzed in this study (in $\mathrm{cm}$ ). The red cell represents the position of the neutron generator

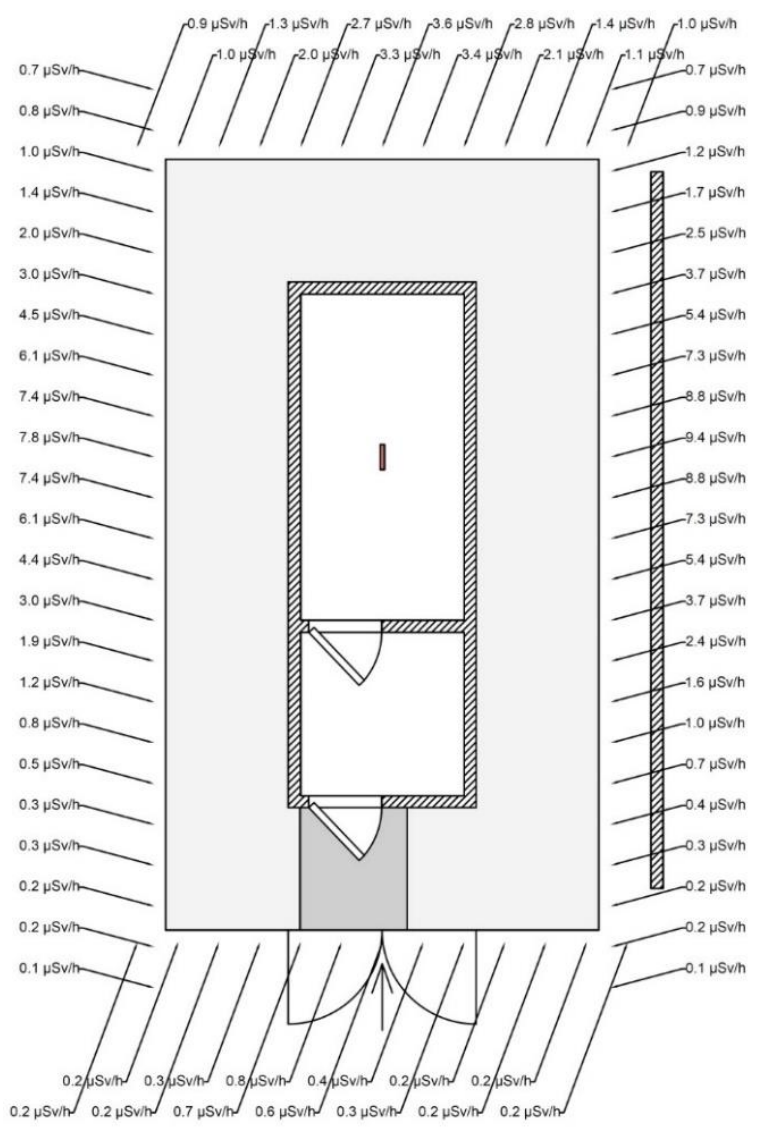

Figure 10. Effective dose rates at $30 \mathrm{~cm}$ from the walls outside the neutron bunker hosting the neutron generator (neutron yield: $10^{8} \mathrm{~s}^{-1}$ ): results from MCNPX simulations
In this case study,

- considering a workload of $200 \mathrm{~h} \mathrm{y}^{-1}$ of the generator operating at maximum neutron yield $\dot{\mathrm{N}}=10^{8} \mathrm{~s}^{-1}$;

- an occupancy factor of $1 / 16$ in the transit area outside the bunker;

- a minimum distance of $5 \mathrm{~m}$ between the generator and the target person from the layout in Figure 9, corresponding to an effective dose rate of $700 \mu \mathrm{Sv} \mathrm{h}{ }^{-1}$ from Figure 8;

- the shielding effect of a single $30 \mathrm{~cm}$ thick concrete slab (that reduces effective dose rates by a factor of 50 at least); the annual effective dose is: $700 \mu \mathrm{Sv} \mathrm{h}^{-1} \cdot 200 \mathrm{~h} \mathrm{y}^{-1} \cdot(1 / 16) \cdot(1 / 50)=175 \mu \mathrm{Sv} \mathrm{y}^{-1}$.

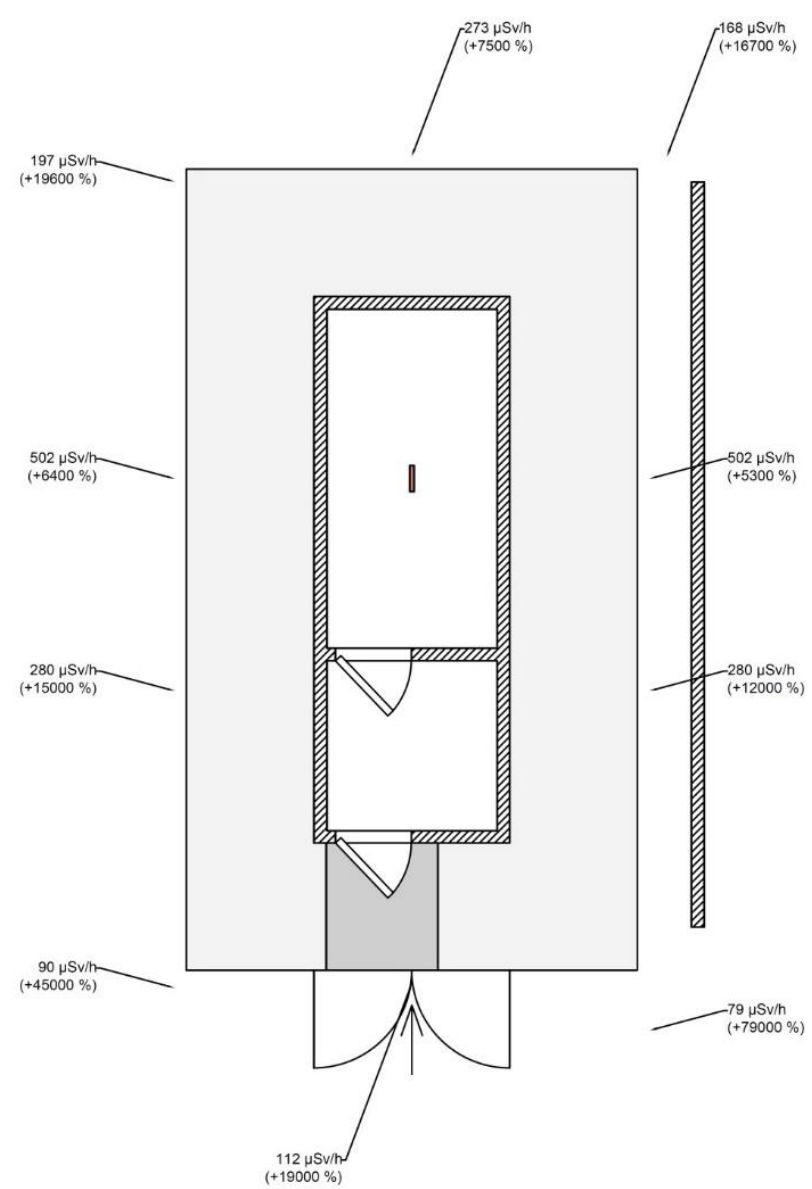

Figure 11. Effective dose rates at $30 \mathrm{~cm}$ from the walls outside the neutron bunker hosting the neutron generator: results from Eq. (2); percentages show the order of magnitude of the overestimations

Regarding the public, annual effective dose from a practice can usually be optimized choosing a criterion between 10 and $300 \mu \mathrm{Sv} \mathrm{y}^{-1}$.

If a member of the public is involved, having chosen 300 $\mu \mathrm{Sv} \mathrm{y}^{-1}$ as optimization criterion, the practice is compliant.

If the $10 \mu \mathrm{Sv} \mathrm{y}^{-1}$ criterion is chosen, the practice is not compliant. In such a case, 1/20 further reduction is needed. Such a reduction can be achieved by one of the following correction actions:

1) reducing the generator's neutron yield from $\dot{\mathrm{N}}=10^{8} \mathrm{~s}^{-1}$ to $\dot{\mathrm{N}}=5 \cdot 10^{6} \mathrm{~s}^{-1}$;

2) reducing the exposure time from $200 \mathrm{~h} \mathrm{y}^{-1}$ to $100 \mathrm{~h} \mathrm{y}^{-1}$ and generator's neutron yield from $\dot{N}=10^{8}$ to $\dot{N}=10^{7} \mathrm{~s}^{-1}$;

3) adding a respect area of $2 \mathrm{~m}$, updating the distance between the generator and the target person to $7 \mathrm{~m}$, 
corresponding to an effective dose rate $\dot{\mathrm{E}}=350 \mu \mathrm{Sv} \mathrm{h}^{-1}$ (from Figure 8); reducing the exposure time from $200 \mathrm{~h} \mathrm{y}^{-}$ ${ }^{1}$ to $100 \mathrm{~h} \mathrm{y}^{-1}$; reducing the neutron generator emission from $\dot{\mathrm{N}}=10^{8}$ to $\dot{\mathrm{N}}=2 \cdot 10^{7} \mathrm{~s}^{-1}$.

\subsubsection{Exposure evaluations in the open-field scenario}

In order to quantify the effective dose conferred to exposed workers and members of the public in case of homeland security applications, a case study regarding the exposure to D-T neutrons in an open-field operation is considered. Such a case study simulates the on-field investigation of an isolated container to check the presence of potential explosive or harmful substances.

The following scenario can be considered:

- $10 \mathrm{~m}$ distance between the control-console and the neutron generator;

- $\quad$ exposed worker engaged for 200 day per year for neutron generator's operations;

- 5 measurement per day i.e. 5 irradiations cycles per day;

- 2 minutes time-span per irradiation to reach the console and switch-on/off the device. It is a conservative assumption due to the fact that neutron generator emission can be scheduled in time by its software programming.

While measurement is running, the operator must wait at a safer position, e.g. behind a shielding wall. In these experimental conditions the total exposure time at $\mathrm{R}=10 \mathrm{~m}$ is $\sim 33 \mathrm{~h} \mathrm{y}^{-1}$. As shown in Figure 8, the effective dose rate at $\mathrm{R}=10$ $\mathrm{m}$ is $\sim 158 \mu \mathrm{Sv} \mathrm{h}{ }^{-1}$, and the effective dose received by the worker is $\sim 5.3 \mathrm{mSv} \mathrm{y}^{-1}$.

Regarding the public, annual effective dose from a practice can usually be optimized choosing a criterion between $10 \mu \mathrm{Sv}$ $\mathrm{y}^{-1}$ and $300 \mu \mathrm{Sv} \mathrm{y}^{-1}$. The neutron generator in the previous scenario is run for 200 day per year, $4 \mathrm{~h}$ per day: using Eq. (2), if the lowest bound $\left(10 \mu \mathrm{Sv}{ }^{-1}\right)$ for the effective dose constraint is selected, from the equation $\dot{\mathrm{E}} \cdot 800 \leq 10 \mu \mathrm{Sv} \mathrm{y}^{-1}$ with $\dot{\mathrm{N}}=10^{8} \mathrm{~s}^{-1}$, an exclusion zone of radius $\mathrm{R}=1200 \mathrm{~m}$ is obtained. If the upper bound for the effective dose constraint is selected, $300 \mu \mathrm{Sv} \mathrm{y}^{-1}$, from the equation $\dot{\mathrm{E}} \cdot 800 \leq 300 \mu \mathrm{Sv}$ $\mathrm{y}^{-1}$ with $\dot{\mathrm{N}}=10^{8} \mathrm{~s}^{-1}$, an exclusion zone of radius $\mathrm{R}=40 \mathrm{~m}$ is obtained.

It should be considered, however, that the approach is conservative, having not accounted for any particular configuration of the environment, i.e. any scattered component. Moreover, it does not involve any shielding structure that may be present in real scenarios, and then the calculated effective dose can actually be interpreted as a maximum value. For instance, the presence of a $30 \mathrm{~cm}$ concrete wall along the 14 $\mathrm{MeV}$ neutrons pathway from the generator to the exposed person may reduce the effective dose rate in Eq. (2) by a factor of 50 at least.

\section{CONCLUSIONS}

The emission of a neutron generator has been simulated in different scenarios related to laboratory and homeland security applications, characterizing the neutron spectra.

The comparison between experimental data and simulated values suggested a procedure for a practical use of the Berthold LB6411 neutron counter as a reliable dosimeter, without the need of former Monte Carlo simulations for calculating the best conversion factors for each specific scenario the dosimeter could be used in. As expected, the operating procedure proved to give conservative results for the evaluation of dose rates.

As an important outcome of the presented experimental campaign validated against Monte Carlo simulations, a standalone and fast calculation tool for planning D-T neutrons experimental exposures was proposed and implemented.

The results of two experimental measurements with a Berthold LB 6411 neutron detector in a neutron bunker were presented, together with the reconstructionist modeling in the MCNPX 2.7.0 virtual world, and validation of simulated data vs experimental data.

Having such model validated, four Monte Carlo simulations with two wider buildings were carried out, to characterize neutron spectral distributions against positioning. The purpose was to evaluate the most appropriate count-rate to dose-rate conversion coefficient for Berthold LB6411.

Results show that the default conversion coefficient for LB6411, calibrated as default with the ${ }^{252} \mathrm{Cf}$ spectrum, should not be used for D-T neutrons exposure evaluation purposes because, in some cases, it would lead to important underestimations. On the contrary, for an adequate, though conservative, estimate, there is evidence that the best solution is to use the conversion coefficient that assumes a monoenergetic neutron spectrum at $14 \mathrm{MeV}$, whatever the environment and geometry of the experimental setup are. Actually, it is worth reminding that in real scenarios neutrons at each point of interest are distributed over the entire energy range, from thermal neutrons $(0.025 \mathrm{eV})$ to $\mathrm{D}-\mathrm{T}$ fusion neutrons $(14.1 \mathrm{MeV})$; the monoenergetic spectrum at $14 \mathrm{MeV}$ is only a conservative assumption to evaluate dose-rates to workers in field scenarios.

Finally, a stand-alone and fast calculation tool for planning experimental exposures was proposed. Such utility tool provides the Radiation Protection Expert with a direct, even though conservative, estimation of the effective dose rate when a neutron generator is operated, taking into account mainly the neutron yield of the device and the distance to the point of interest. The tool assumes the sole presence of air between the neutron generator and the target person at the point of interest. However, any presence of a shielding structure can be taken into account by a scaling factor to be applied to effective dose rates.

Two case-studies were discussed and analyzed as examples of evaluation of the effective dose-rates to exposed workers and members of the public:

1) an "open-field" geometry without any assumptions on setup, shielding slabs or moderating structures, was considered to propose an effective dose rate evaluation approach, useful for a priori exposure assessments. Calculated dose rates could be intended as maximum values, being the approach as general and conservative as possible;

2) a "bunker-scenario" was then considered, approaching the issue by means of Monte Carlo MCNPX calculations for preliminary estimation of dose rates. A comparison with the results previously discussed shows the conservativeness of the simplified model in Eq. (2).

Given the non-specificity of the considered scenarios, these conclusions are general in nature. The method was developed for first, rough but conservative, exposure evaluations under environmental conditions whose effects on neutrons spectra at the point of interest are not a priori predictable, i.e. complex irradiation scenarios on field, as a shipping port, research facilities, etc. The proposed approach simplifies the 
assessment, suggesting a procedure for obtaining conservative results without the need of time-expensive Monte Carlo simulations.

\section{ACKNOWLEDGMENT}

The authors would like to express special thanks to Dr. Mario Pillon (ENEA) for his disposability in dialogue and his technical competences in discussing the radiographic data of the Thermo Scientific MP-320 neutron generator.

Thanks go to the Institute of Radiation Protection of ENEA for providing the calibrated Berthold LB6411 neutron counter used in the experimental campaigns.

A special thanks goes to all ENEA-UTICT technicians working on HPC system used in this work. The computing resources and the related technical support were provided by the CRESCO/ENEAGRID High Performance Computing infrastructure and its staff [23]. CRESCO/ENEAGRID High Performance Computing infrastructure is funded by ENEA and by Italian and European research programs, see http://www.cresco.enea.it/english for information.

\section{REFERENCES}

[1] Csikai, G.J. (1987). CRC Handbook of Fast Neutron Generators, vol. 1. Boca Raton, Florida, United States: CRC Press.

[2] Raoux, A.C., Lyoussi, A., Passard, C., Denis, C., Loridon, J., Misraki, J., Chany, P. (2003) Delayed neutron measurement, Prompt neutron measurement, Waste characterisation, Monte Carlo uncertainties. Nuclear Instruments and Methods in Physics Research Section B: Beam Interactions with Materials and Atoms, 207(2): 186-194. https://doi.org/10.1016/S0168$583 X(03) 00451-8$

[3] Gozani, T. (1981). Active nondestructive assay of nuclear materials: Principles and applications. Washington, D.C., United States, U.S. Nuclear Regulatory Commission, NUREG/CR-0602.

[4] Buffler, A. (2004). Contraband detection with fast neutrons. Radiation Physics and Chemistry, 71(3-4): 853-861.

https://doi.org/10.1016/j.radphyschem.2004.04.110

[5] Aleksandrov, V.D., Bogolubov, E.P., Bochkarev, O.V., Korytko, L.A., Nazarov, V.I., Polkanov, Y.G., Ryzhkov V.I, Khasaev, T.O. (2005). Application of neutron generators for high explosives, toxic agents and fissile material detection. Applied Radiation and Isotopes, 63(56):

$537-543$

https://doi.org/10.1016/j.apradiso.2005.05.002

[6] Bogolubov, Y.P., Korotkov, S.A., Korytko, L.A., Morukov, V.G., Nazarov, V.I., Polkanov, Y.G., Khasaev, T.O. (2004). Method and system based on pulsed neutron generator for fissile material detection in luggage. Nuclear Instruments and Methods in Physics Research Section B: Beam Interactions with Materials and Atoms, 213: 439-444. https://doi.org/10.1016/S0168$583 \mathrm{X}(03) 01751-8$

[7] Cherubini, N., Dodaro, A., Gandolfo, G., Lepore, L., Marzo, G.A., Piccinelli, E., Remetti, R. (2016). Field prototype of the ENEA neutron active interrogation device for the detection of dirty bombs. Challenges, 7(2):
17. https://doi.org/10.3390/challe7020017

[8] Remetti, R., Gandolfo, G., Lepore, L., Cherubini, N. (2017). In field application of differential Die-Away time technique for detecting gram quantities of fissile materials. Nuclear Instruments and Methods in Physics Research Section A: Accelerators, Spectrometers, Detectors and Associated Equipment, 870: 123-130. https://doi.org/10.1016/j.nima.2017.07.033

[9] Jordan, K.A., Gozani, T. (2007). Pulsed neutron differential die away analysis for detection of nuclear materials. Nuclear Instruments and Methods in Physics Research Section B: Beam Interactions with Materials and Atoms, 261(1-2): 365-368. https://doi.org/10.1016/j.nimb.2007.04.294

[10] Jordan, K.A., Gozani, T., Vujic, J. (2008). Differential die-away analysis system response modeling and detector design. Nuclear Instruments and Methods in Physics Research Section A: Accelerators, Spectrometers, Detectors and Associated Equipment, 589(3): 436-444 https://doi.org/10.1016/j.nima.2008.02.039

[11] Vourvopoulos, G., Womble, P.C. (2001). Pulsed fast/thermal neutron analysis: A technique for explosives detection. Talanta, 54(3): 459-468. https://doi.org/10.1016/S0039-9140(00)00544-0

[12] Andersson, I.O. (1963). Neutron rem-counter with uniform sensitivity from $0.025 \mathrm{eV}$ to $10 \mathrm{MeV}$. In Proc. IAEA Symp. on Neutron Dosimetry, Vienna, 2: 87-95.

[13] Birattari, C., Ferrari, A., Nuccetelli, C., Pelliccioni, M. Silari, M. (1990). An extended range neutron rem counter. Nuclear Instruments and Methods in Physics Research Section A: Accelerators, Spectrometers, Detectors and Associated Equipment, 297(1-2): 250-257. https://doi.org/10.1016/0168-9002(90)91373-J

[14] Jagerhofer, L., Feldbaum, E., Forkel-Wirth, D., Theis, C., Vincke, H., Iwamoto, Y., Hagiwara, M., Satoh, D., Iwase, H., Yashima, H., Matsumoto, T., Mmasuda, A., Nishiyama, J., Nakamura, T., Sato, T., Nakane, Y., Nakashima, H., Sakamoto, Y., Tamii, A., Hatanaka K. (2011). Characterization of the WENDI-II REM counter for its application at MedAustron. Prog. Nucl. Sci. Technol, 2: 258-262.

[15] Jägerhofer, L., Feldbaumer, E., Theis, C., Roesler, S., Vincke, H. (2012). A new method to calculate the response of the WENDI-II rem counter using the FLUKA Monte Carlo Code. Nuclear Instruments and Methods in Physics Research Section A: Accelerators, Spectrometers, Detectors and Associated Equipment, 691: 81-85. https://doi.org/10.1016/j.nima.2012.05.097

[16] Kim, S.I., Kim, B.H., Chang, I., Lee, J.I., Kim, J.L., Pradhan, A.S. (2013). Response of six neutron survey meters in mixed fields of fast and thermal neutrons. Radiation Protection Dosimetry, 156(4): 518-524. https://doi.org/10.1093/rpd/nct103

[17] Schwartz, R.B., Eisenhauer, C.M. (1979). The design and construction of a $\mathrm{D}_{2} \mathrm{O}$-Moderated ${ }^{252} \mathrm{Cf}$ source for calibrating neutron personnel dosimeters used at nuclear power reactors. US Nucl Reg Com Doc. 1980 Jan. NUREG/CR-1204.

[18] Contessa, G.M., Cherubini, N., Dodaro, A., Lepore, L., Marzo, G.A., Sandri, S. (2015). Caratterizzazione teorica e sperimentale del fascio neutronico di un generatore di neutroni del tipo D-T e conseguente analisi dosimetrica Theoretical and experimental characterization of the 
neutron beam of a D-T neutron generator and consequent dosimetric analysis. Proceedings of XXXVI National Congress of Radiation Protection - Italian Radiation Protection Association. https://www.airpasso.it/?page_id=731.

[19] MP320 Neutron Generator - Operation Manual. (2014). Thermo Fisher Scientific, Waltham, USA. https://assets.thermofisher.com/TFSAssets/CAD/Specification-Sheets/D10497 .pdf.

[20] LB6411 Neutron Probe - Operation Manual. (1995). Berthold Technologies GmbH, Vienna, Austria. https://www.berthold.com/en/radiationprotection/products/dose-and-dose-rate/neutron-doserate-probe-lb-6411/.

[21] Briesmeister, J.F. (1993). MCNP-A general purpose Monte Carlo code for neutron and photon transport.
Manual Version C, 4.

[22] Petoussi-Henss, N., Bolch, W.E., Eckerman, K.F., Endo, A., Hertel, N., Hunt, J., Pelliccioni, M., Schlattl, H., Zankl, M. (2010). Conversion coefficients for radiological protection quantities for external radiation exposures. Annals of the ICRP, 40(2-5): 1-257. https://doi.org/10.1016/j.icrp.2011.10.001

[23] Iannone, F., Ambrosino, F., Bracco, G., De Rosa, M., Funel, A., Guarnieri, G., Migliori, S., Palombi, F., Ponti, G., Santomauro, G., Procacci, P. (2019). CRESCO ENEA HPC clusters: a working example of a multifabric GPFS Spectrum Scale layout. In 2019 International Conference on High Performance Computing \& Simulation (HPCS), 1051-1052. https://doi.org/10.1109/HPCS48598.2019.9188135 